Psychosomatic Medicine 72:656-663 (2010). DOI: 10.1097/PSY.0b013e3181ec5373

\title{
Randomized Controlled Two-Stage Trial in the Treatment of Bulimia Nervosa, Comparing CBT Versus Motivational Enhancement in Phase 1 Followed by Group Versus Individual CBT in Phase 2
}

MELANIE A. KATZMAN, PHD, NURIA BARA-CARRIL, MSC, SOPHIA RABEHESKETH, PHD, ULRIKE SCHMIDT, MD, PHD, MRCPSYCH, NICHOLAS TROOP, PHD, AND JANET TREASURE, PHD, FRCP, FRCPSYCH

From the Department of Psychiatry (M.A.K.), Weill Cornell Medical Center, New York, New York; Eating Disorders Unit (N.B.-C.), Maudsley Hospital, London, United Kingdom; Graduate School of Education (S.R.-H.), University of California, Berkeley, Berkeley, California, and University of London, Institute of Education, London, United Kingdom; Section of Eating Disorders (U.S., J.T.), Institute of Psychiatry, London, United Kingdom; and the Department of Psychology (N.T.), London Metropolitan University, London, United Kingdom.

Address correspondence and reprint requests to Melanie A. Katzman, 10 East 78th Street, Suite 4A, New York, NY 10075. E-mail: mkatzman@katzmanconsulting.com Received for publication July 2, 2009; revision received May 10, 2010. The authors have not disclosed any potential conflicts of interest. 


\begin{abstract}
Objective: To conduct a randomized, controlled, two-stage trial in the treatment of bulimia nervosa, comparing cognitive behavioural therapy (CBT) versus motivational enhancement in Phase 1 followed by group versus individual CBT in Phase 2.

Methods: A total of 225 patients with bulimia nervosa or eating disorder not otherwise specified (EDNOS) were recruited into a randomized controlled trial lasting 12 weeks with follow-ups at 1 year and 2.5 years. Results: Patients improved significantly across all of the interventions with no differences in outcome or treatment adherence. Including motivational enhancement therapy rather than a CBT first phase of treatment did not affect outcome.

Conclusions: Outcome differences between individual and group CBT were minor, suggesting that group treatment prefaced by a short individual intervention may be a costeffective alternative to purely individual treatment.
\end{abstract}

Key words: bulimia nervosa, group cognitive-behavioral therapy, motivational enhancement therapy, randomized controlled trial. 


\section{INTRODUCTION}

The National Institute for Clinical Excellence Guidelines for Eating Disorders (1)

recommend that a specific form of cognitive-behavior therapy for bulimia nervosa (CBT-BN)

(2) should be given to most adults. The statement was based on evidence from randomized controlled trials, which generally assess the efficacy of treatment under optimal conditions (highly selected patients, recruited via advertisements and treatment delivered by research clinicians with tightly controlled treatment procedures). Many patients presenting to outpatient services do not have full syndromal bulimia nervosa $(\mathrm{BN})$ and instead present with subthreshold or partial syndromes (3)' very few studies (4 -7) of reasonable size (i.e., 100 participants) have included these patients. Thus, little is known about the effectiveness of CBT-BN under routine clinical conditions (8), where treatments are delivered by ordinary therapists to patients with symptoms that do not neatly fit the diagnostic category of BN (i.e., those with eating disorder not otherwise specified [EDNOS]).

Recent efficacy studies (9-11), which have compared group with individual CBT, suggested a slight advantage of individual over group treatment in terms of clinical outcomes, in part, because there is less adherence to group treatment. In routine clinical practice, dropout from individual CBT is high (approximately $40 \%$ to $50 \%$ ), whether delivered individually or in a group setting $(12,13)$. The failure to adhere to a treatment with proven effectiveness suggests that there may be a problem in readiness to change. Motivational interviewing is an intervention, which was developed to address just such concerns, and when offered as a prelude to other forms of psychological treatment, such as CBT, has produced synergistic effects (14). Motivational interventions given in the first phase of treatment for people with eating disorders have been found to improve outcome $(15,16)$ and increase readiness to 
change (15). The addition of a Motivational Interviewing component as a prelude to group treatment might augment the commitment to behavior change.

The present study reflects a pragmatic clinical trial under routine conditions in a busy outpatient setting, to examine whether a) motivational enhancement therapy (MET) before individual (MET-I) or group delivery of CBT (MET-G) will reduce dropout and improve outcome in patients with BN or EDNOS compared with participants who received purely CBT both before and during group care (CBT-G); and b) whether group treatment (MET-G and CBT-G) can produce similar clinical outcomes compared with a purely individual treatment of the same duration (MET-I).

\section{METHODS}

\section{$\underline{\text { Subjects }}$}

Patients were recruited from consecutive primary care or secondary care referrals to a catchment area-based NHS eating disorder service. All patients fulfilling the Diagnostic and Statistical Manual of Mental Disorders, Fourth Edition criteria for BN or EDNOS were eligible for the study. The exclusion criteria were pregnancy, diabetes mellitus, severe mental illness (such as schizophrenia or bipolar illness), severe learning disability, inability to commit to treatment from the outset, or referral for assessment only. We defined EDNOS as subthreshold $\mathrm{BN}$ - a clinically relevant eating disorder (i.e., significant impairment of physical health or psychosocial functioning) where the patient met the criteria for BN except that the binge eating and/or inappropriate compensatory behaviors occurred at a frequency of less than twice a week or for a duration of 3 months. In our sample of 225, 60 were diagnosed with EDNOS. The study was approved by the Ethics 
Committee of the Institute of Psychiatry and South London and Maudsley NHS Trust and took place over a 4.5-year period, beginning late 1997 and ending in 2002.

\section{Sample Size}

The sample size calculation took into account a difference that might be considered to be clinically significant between groups in this population. We calculated that a sample size of 64 patients per treatment condition was required to provide $80 \%$ power at two-sided $\mathrm{p}<.05$ to detect a difference of .5 in the size of the effect of change in symptom frequency. To account for dropout, we increased the sample to 75 per group.

\section{$\underline{\text { Randomization }}$}

An independent investigator, using a table of random numbers, generated the randomization sequence. Allocations were contained in sequentially numbered, sealed, opaque envelopes opened by the clinician after the initial assessment, during which eligibility and willingness to participate were determined.

\section{$\underline{\text { Study Design }}$}

A three-group, two-phase study design was adopted with patients being allocated to either four sessions of individual MET followed by eight sessions of individual CBT (MET-I), four sessions of individual MET followed by eight sessions group CBT (MET-G), or four sessions of individual CBT followed by eight sessions of group CBT (CBT-G).

\section{Phase 1}

Phase 1 consisted of four individual sessions of either manualized individual MET or individual CBT for BN. 
MET

In MET, the therapist used principles of motivational interviewing and accompanying worksheets guided by the manual "A Clinician's Guide to Getting Better Bit(e) by Bit(e)" (17). Treatment focused on a consideration of the benefits of changing and the barriers to be overcome to change, moving from the here and now by envisioning key values and how these would fit into the whole life story. No unsolicited advice about eating was given. All participants in this condition received a letter providing personalized feedback on physical symptoms, laboratory tests, and detailed social, family, educational, and vocational problems found at the time of assessment. This feedback was reviewed by patient and therapist during the first session. Behavior change techniques included: outcome expectancies, personal relevance, descriptive norms, developing personal and moral norms and if the patient showed a commitment to change, concrete planning and contracting of behavioral goals (17).

\section{CBT}

In the CBT condition, therapists followed the instructions of the first four chapters of "bulimia nervosa" (18) and included active strategies of behaviour change from session 1, including nutritional/food monitoring sheets, meal planning, activity lists, and problemsolving activities. At the time of the study, this was the only CBT self-help manual available with proven efficacy in the treatment of $\mathrm{BN}$. This manual does not specifically focus on increasing motivation.

\section{Phase 2}

Phase 2 consisted of eight sessions of individual or group CBT. In both conditions, patients worked with the manual, "You Can't Have Your Cake and Eat It Too: A Progam for 
Controlling Bulimia" (19). The structure of Phase 2 sessions followed the chapter topics of the book. Each week included discussion and exercises to educate women about the physical and psychological hazards of eating disorders, challenging myths, and identifying ways women might undermine their own success. During weekly sessions, therapists integrated nutritional information (realistic caloric consumption, meal planning, etc.) and methods to modify extreme, unhelpful thinking. Perfectionist ideas about one's body and behavior were identified and ways to assert one's feelings and express anger were reviewed. Women discussed basic human rights; obstacles to feeling satisfied in their relationships and evaluated which relationships are "nourishing." They engaged in role plays to tackle interpersonal problems and were encouraged to use the group to test new behaviors. Women identified media expectations for appearance and brought in pictures of their real and ideal body. Exercises included ways to appreciate the function, not the form of their body, and participants experimented with making changes in attractive behaviors, rather than weight. Everyone kept a diary, which was collected each week and returned the next week with written suggestions and encouragement by the therapist. Progress was recorded each week on a standing flip chart (if in group) or on a graph (if in individual care), and any movement toward decreasing or delaying binges was recognized. The flip chart was also used to capture the work of each exercise and was reviewed by participants over the course of the group as different activities referenced back to each other. The chart was also used during supervision. As the group progressed, the diary work became increasingly focused on aiding women to use the I.C.O.P.E. method (20) which involved: Identifying the problem, Communicating the issues more assertively, Organizing one's time for maximum benefit-setting limits, Perceiving things realistically and positively (whether it be their bodies or their behaviors), and Enhancing one's self-esteem through nourishing actions and relationships. Individual sessions lasted 50 minutes. Group sessions lasted 90 minutes, had between six to eight 
participants, and were moderated by two therapists. To ensure continuity, therapists running groups also saw patients for Phase 1 treatments so that each group participant had one of the two co-leaders as their therapist during the first four sessions. Both co-leaders played equal roles.

In contrast to the well-known CBT-BN program of Fairburn and colleagues (2), the group treatment used here $(19,21)$ is briefer ( 8 versus 19 sessions) and emphasized women's development of interpersonal competencies.

\section{Therapist Training and Supervision}

Therapists were all drawn from health service personnel in the Eating Disorders Unit at the Maudsley Hospital and included psychologists, psychiatrists, nurses, or occupational therapists, all of whom were experienced in the treatment of eating disorders and the delivery of MET and CBT. To avoid therapist bias, all therapists delivered the three conditions. All received the following: MET training during a 2-day workshop; a training day on the use of the group program; and a 2-day workshop on the delivery of manual-guided CBT. Weekly supervision was mandatory for all therapists and was conducted on a one:one basis for individual treatment and with co-therapists for the group intervention. Supervision arrangements for the first 4 weeks of treatment were kept strictly separate and conducted by separate supervisors who were either experienced CBT (Padmal de Silva) or MET trainers (J.T., U.S.). Dr. Katzman provided supervision to all three experimental groups during the last 8 weeks of the trial, again with separate supervision for cases being treated in individual or group treatment. During the first 4 weeks, all treatment conditions (MET-I, MET-G, CBTG) generated a "product" (thought records, motivational letters, etc). These were checked for quality in supervision. Moreover, precisely how materials were used in therapy and integrated 
into the treatment of different patients was reviewed in supervision on a regular basis. In Phase 2, all diaries and flip charts were collected weekly and reviewed by supervisors.

\section{$\underline{\text { Assessment }}$}

All patients were assessed for suitability by an experienced clinician, using a standardized semi-structured interview format (22-24). The English version of the interview was developed by eating disorder experts in the context of a European project (COST ACTION B6). Previous versions of the interview had been used in a large German multi-center, naturalistic study on outcome of eating disorders (project TREAT) (22). The interview included variables from the LIFE (24) and the EDE (23) assessing eating disorder symptoms, use of drugs, and alcohol and antidepressant medication. A pre-treatment battery (22) included basic clinical features and demographics, along with well-validated, widely used questionnaires, such as the Symptom Checklist 90 (25). The primary treatment outcomes were key behavioral symptoms (binge eating, self-induced vomiting, laxative/diuretics abuse) that were measured, using the short evaluation of eating disorders $(26-28)$. This is a brief, valid, and reliable self-report measure assessing eating disorder symptoms over the last 4 weeks. There is excellent interrater reliability with values of $>0.70$ between patient selfratings and those independently made by an "expert" (therapist or clinical researcher). Patients and therapists rated these variables separately at pretreatment, 4 weeks (after Phase 1) and at 12 weeks (after Phase 2). Only patient ratings collected by research assistants blinded to their treatment allocation are available for the 1-year follow-up and the 2.5-year follow-up. All outcomes were assessed for the time period of the previous month.

An additional outcome variable, motivation to change and dropout, was assessed with the University of Rhode Island, Change Assessment Scale (29). This questionnaire, completed by 
the patient, offers four scales - precontemplation, contemplation, action, and maintenanceas well as assigning patients to a particular stage on the basis of the scale with the highest score.

\section{$\underline{\text { Definitions of Treatment Completion and Dropout }}$}

There is little agreement between studies on how to define treatment adherence and dropout. We used completion of treatment phases as a proxy of treatment adherence. Phase 1 treatment (MET or CBT) completion was defined as attendance at all four individual sessions. Completion of Phase 2 treatment (individual or group CBT) was defined as attendance at $\geq 6$ of eight possible sessions. Dropout was defined as Phase 1 completed and Phase 2 not completed or non-completion of Phase 1 and Phase 2 treatment.

\section{$\underline{\text { Analysis }}$}

Demographic information and baseline data were analyzed, using SPSS 8.0 (SPSS, Inc., Chicago, Illinois) for windows. Data were compared using t-tests, analysis of variance, or $\chi^{2}$. Eating disorder symptomatology was analyzed, using the Stata program gllamm (30). For this, analysis outcomes for eating disorder symptoms (bingeing, vomiting, laxative use) were examined separately to determine whether motivational pre-treatment might have a different effect on different symptoms (given that clinical experience suggests that patients' motivation to change differs between symptoms).

The patient and therapist ratings of the frequency of bingeing, vomiting, and use of laxatives were collapsed into three comparable and clinically relevant categories: $0=$ abstinence; $1=$ infrequent behaviors (i.e., $<2$ times a week); and $2=$ frequent behaviors $(\geq 2$ times a week). 
Therapist and patient ratings of each type of behavior were analyzed separately. A proportional odds model for repeated measures was used to model the effect of treatment, time, and time by treatment on the odds of being rated in the higher categories ( 2 versus 1,0 or 2, 1 versus 0 ). A random effect for subjects was included in the model to take account of correlations among the observations on the same subject. The effect of time was modeled by a linear trend in the log-odds. The significance of the interaction was tested, using a Wald test. If significant, the linear trend of time was compared between the groups with and without motivational enhancement therapy and between the group treatment and individual treatment groups. If the group by time interaction was not significant, the model was reestimated with the main effects of group and time only.

Our analyses were based on intention to treat, which meant that although each subject contributed different numbers of observations, we were able to make more efficient use of the data than the more common complete case analysis used for repeated-measures data. This approach also allowed us to analyze whether specific variables, such as dropout or stage of change, predicted outcome. The parameters were estimated by maximum likelihood, using adaptive quadrature. Maximum likelihood estimation provides consistent parameter estimates if the data are missing at random.

\section{RESULTS}

$\underline{\text { Recruitment and Progression of Patients Through the Study }}$

Figure 1 provides a CONSORT diagram of patient recruitment and their progression through the study. 


\section{Baseline Data}

As shown in Table 1, analysis of variance revealed no significant differences between any of the three groups on baseline characteristics apart from a difference in age, which although statistically significant $(\mathrm{p}<.05)$ was not clinically meaningful (mean age, 29.3 years; MET-I, 31.0 years; MET-G, 28.9 years; CBT-G, 27.8 years). For the groups combined, the average body mass index $\left(\mathrm{kg} / \mathrm{m}^{2}\right)$ was $24.7(7.5)$.

\section{Table 1 about here}

The three groups reported no difference at baseline on bingeing, vomiting, or laxative use. These variables were rated, using a 5 -point scale (" $1=$ not at all," " $2=$ up to $1 \times$ per week," "3 $=2$ to $3 \times$ per week," " $4=4 \times$ per week up to daily," " $5=>1 \times$ day"). It is of note that considerable proportions of patients took antidepressant medication, (42.4\% of the 172 patients for whom this information was available), used alcohol regularly (51.5\% of the 165 patients for whom this information was available) or were taking illicit drugs (42.1\% of the 155 patients for whom this information was available). The participants drank between 4 times a week to daily (10\% drank more than once daily). This suggests high levels of comorbid substance abuse and depression, which did not differ between groups (Pearson's $\chi^{2}$ $=1.306, \mathrm{p}=.520$ when comparing groups on use of antidepressant medication; Pearson's $\chi^{2}$ $=15.290, \mathrm{p}=.054$ comparing groups on alcohol use and Pearson's $\chi^{2}$ of $8.698, \mathrm{p}=.368$ on illicit drug use). 
There were no significant differences in treatment uptake, completion, and dropout between groups (Fig. 2). Patient ratings of symptom abstinence beginning to end of treatment are illustrated in Table 2.

\section{Figure 2 about here}

\section{Table 2 about here}

\section{Bingeing}

\section{Therapist Rating}

There was no significant group by time interaction $(\mathrm{p}=.79)$. The odds of bingeing decreased over time $(\mathrm{p}<.001$; odds ratio [OR], 0.32; 95\% confidence interval [CI], 0.19-0.53). Stage of change, Symptom Checklist-90 (SCL-90), and completion of Phase 1 had no significant effect on outcome.

\section{Patient Rating}

There was no significant group by time interaction $(p=.64)$. The reduction in the odds of bingeing over time was significant ( $<$.001; OR, 0.39; 95\% CI, 0.31-0.49). The patient rating of bingeing over time tended to be lower if the patient was in a higher stage of change at the time of assessment $(\mathrm{p}=.02)$, i.e., more motivated. The SCL-90 and completion of Phase 1 had no significant effect on the patient ratings of bingeing.

\section{Self-Induced Vomiting}

\section{Therapist Rating}

Vomiting as rated by the therapist did not show a significant group by time interaction $(\mathrm{p}=$ .62). There was a significant main effect of time. At every assessment, the odds of vomiting 
decreased to $36 \%$ of its value at the previous assessment $(\mathrm{p}<.001$; OR, 0.36 ; 95\% CI, $0.25-$ 0.53). Completion of the first treatment phase significantly reduced the odds of vomiting $(\mathrm{OR}, 0.28 ; \mathrm{p}=.05 ; 95 \% \mathrm{CI}, 0.08-1.01)$. Scores on the SCL-90, stage of change and dropout had no significant effect.

\section{Patient Rating}

The patient rating showed no significant group by time interaction $(p=.61)$. The odds of vomiting decreased significantly over time ( $\mathrm{p}<.001$; OR, 0.46; 95\% CI, 0.36-0.58). Completion of the first treatment phase significantly reduced the odds of vomiting (OR, 0.29; $\mathrm{p}=.05 ; 95 \%$ CI, 0.09-0.99). SCL-90 scores and stage of change had no significant effect on outcome.

\section{$\underline{\text { Laxative or Diuretic Use }}$}

\section{Therapist Rating}

The therapist rating of laxative/diuretic use showed a significant group by time interaction ( $\mathrm{p}$ $=.03)$. The effect of time differed significantly between the condition which received MET-G and the condition which received MET-I $(\mathrm{p}=.01)$ and not between any other pairs. The effect of time was not significant in the MET-G condition $(\mathrm{p}=.21)$ but was significant in the CBT$\mathrm{G}(\mathrm{p}=.01 ; \mathrm{OR}, 0.39 ; 95 \% \mathrm{CI}, 0.19-0.81)$ and in the MET-I condition $(\mathrm{p}<.001 ;$ OR, 0.15; 95\% CI, 0.06-0.42). The two conditions which had group treatment in Phase 2 differed significantly from the condition which had individual treatment in Phase $2(\mathrm{p}=.03)$ but the conditions which had MET in Phase 1 did not differ significantly from the condition which had CBT throughout. The odds of laxative use were greater in the group that received group therapy. 
Completion of Phase I, SCL-90 and stage of change had no significant effect on the therapist rating of laxative use.

\section{Patient Rating}

The patient rating of laxative/diuretic use showed no significant group by time interaction ( $\mathrm{p}$ $=.45)$. There was a significant main effect of time, showing that the odds of laxative/diuretic use decreased ( $\mathrm{p}<.001 ;$ OR, 0.53; 95\% CI, 0.41-0.70). The SCL-90 score predicted the odds of laxative/diuretic usage as judged by the patient $(\mathrm{p}=.006$; OR, 4.22, 95\% CI, 1.511.86), but completion of Phase 1 and stage of change had no significant effect.

\section{$\underline{\text { Abstinence Rates }}$}

Table 3 illustrates abstinence rates for bulimic behaviors over time as rated by the patients and therapists.

\section{Table 3 about here}

Given the lack of differences between the three groups on the key behavioral variables, overall abstinence rates were calculated for patient and therapist. For binge eating, the overall abstinence rates on the therapist assessment were $12.5 \%$ at baseline and $46 \%$ at post treatment. Patient rating of bingeing abstinence was $2.5 \%$ at baseline, $29 \%$ at post treatment, $40 \%$ at 1 -year follow-up, and $46 \%$ at 2.5 -year follow-up.

For self-induced vomiting, the overall abstinence rate by therapists was $23 \%$ at baseline and $45 \%$ at post treatment. Patient rating of self-induced vomiting was $21 \%$ at baseline, $29 \%$ at post treatment, $54 \%$ at 1 -year follow-up, and $46 \%$ at 2.5 -year follow-up. 
For abstinence from laxative/diuretic abuse, therapists rated $67 \%$ at baseline and $87 \%$ at post treatment. Patients reported abstinence rates of $59 \%$ at baseline, $75.3 \%$ at post-treatment, $80 \%$ at 1-year follow-up, and $85 \%$ at 2.5 -year follow-up.

\section{DISCUSSION}

This is a large-scale, pragmatic catchment area-based investigation of treatment effectiveness for bulimic disorders (BN and EDNOS).

\section{Main Findings}

Patients in all three conditions improved significantly on key behavioral outcomes (frequency of bingeing, vomiting, and laxative abuse). The degree of improvement on these parameters is in keeping with those found in other studies $(1,31)$. It is noteworthy that patients reported more binge eating than therapists - perhaps because the patients themselves do not distinguish between subjective and objective binges.

There were no differences between conditions on bingeing or vomiting (whether assessed by the patient or the therapist). While on the therapist rating of laxative abuse there was a significant group by time interaction, the patient rating of laxative use failed to confirm this. As only a small proportion of patients used laxatives, overall there is less certainty about this finding.

There were no differences between groups in treatment up-take, completion, or dropout rates. The dropout rates found here, although higher than those reported in efficacy studies, were 
comparable to those reported in the context of other sample, using individual or group CBT in routine clinical settings $(11,32)$.

MET Versus CBT in the First Treatment Phase Our first hypothesis was that MET would improve treatment adherence and reduce dropout from treatment in patients with BN. This hypothesis was not supported. However, our findings do suggest that MET constitutes an acceptable alternative to CBT early in treatment, which is of both theoretical and practical importance. Theoretically, this is significant because MET involves different processes to CBT (33); the latter, for example, uses self-monitoring among other forms of actionorientated behavior change principles in the early stages of treatment. It has been found that the response to CBT in the first six sessions is a strong predictor of later outcome (34). Those patients who find it difficult to comply with active strategies may respond better to MET. The majority of patients in this study were in the contemplation stage at baseline, and none were in precontemplation (35). Motivational interviewing is a less useful intervention in people who are ready to change (36). It is possible that the use of MET may be shown to be more effective in improving adherence and subsequent outcome in a less motivated group than the current sample who had sought clinical help.

\section{Group Versus Individual Treatment}

Our second hypothesis was that adding a brief individual component to group treatment can produce comparable results to solely individual treatment of the same duration. Patients in our study did equally well whether treated individually throughout or in an individual plus group setting in Phase 2. This finding counters past research, which suggests that group treatment produces higher dropout rates and may be less effective in reducing key behaviors, such as self-induced vomiting (37). 
It is possible that our paper was underpowered to find statistical significance in abstinence rates between the group and individual conditions. However, it is worth considering several (not mutually exclusive) possibilities as to why the group worked as well (if not better) than the individual treatment program. These offer possible areas for future, more definitive research assessing group versus individual care. The short individualized prelude may have better prepared them for the group. Also, the therapist who saw the patient in the first phase of treatment was always one of the two therapists running the group to which the patient was allocated. This continuity and the patient's knowledge that the therapist knew this person as an individual may have produced more positive outcomes. These optimistic findings for the use of group relative to individual care parallel those of Chen et al. (9), even though the programs were of different length and content.

It is also notable that the length of our group treatment was 8 weeks, which is shorter than other group interventions described in the literature. However, Wolchik et al. (21) reported clinically significant findings after 7 weeks, and other investigators have reported that most change happens in the early phase of care (34).

Overall, our findings suggest that, in routine clinical practice, with a few individual sessions at the start, group treatment is a valid and useful alternative to individual therapy. Importantly, group treatment may have advantages in terms of cost-effectiveness. In our setting, the total therapist time for one patient treated individually throughout was approximately 12 hours, whereas the therapist time for one patient treated in a group after four individual sessions was 8 hours. Obviously, more detailed costeffectiveness assessments are needed. 


\section{Predictors of Outcome}

We used psychiatric comorbidity as measured by the SCL-90, pre-treatment motivation (assessed by the stage of change measure, University of Rhode Island, Change Assessment Scale), and completion of the first treatment phase as predictors of outcome. These predictors were chosen because of their demonstrated importance in previous studies $(34,35)$.

Completion of the first treatment phase was the only variable to predict outcome on both patient and therapist assessment, and it only predicted outcome from vomiting. This is noteworthy, as early reduction of vomiting is an important predictor of longer-term treatment success (34). In the current study, long-term reduction in binge eating was predicted by the initial level of motivation. This supports earlier findings by Treasure et al. (35) in which improvement in binge eating was found for those subjects who shifted into an "active" stage of change. This also suggests that the stage of change may be specific for each of the behaviors (bingeing, vomiting, laxative abuse) and, therefore, should be assessed independently. That different behaviors may respond differently to treatment may advocate for interventions organized around symptoms, rather than diagnoses as suggested by the transdiagnostic model of Fairburn and colleagues $(7,38)$.

\section{$\underline{\text { Limitations }}$}

Our study was conducted with minimal resources under routine clinical conditions and, as a result, has a number of methodological limitations. Due to resource constraints, we did not include the "obvious" fourth treatment cell (four sessions CBT followed by eight sessions individual CBT). The inclusion of such a group would have been desirable, as it would have allowed us to assess whether MET enhances outcome relative to CBT, irrespective of 
whether it is followed by individual or group treatment. We also did not include a notreatment control group. This was neither practical nor given the pragmatic nature of the trial critical to understanding our results, as past studies, including one from our own center, did not show spontaneous improvement in the waiting list condition (39).

We used patient ratings only for the long-term follow-up in keeping with previous large-scale naturalistic eating disorder outcome studies $(27,28)$ Previous studies $(40)$ have demonstrated excellent agreement between patient self-assessment tools and expert ratings on self-induced vomiting and on laxative use with lesser agreement on variables, such as bingeing, which require more subjective judgment. This is exactly what we found in the present studypatient/clinician agreement on symptom frequency was better for vomiting and laxative use than for bingeing. However, overall, the inter-rater reliability between patients and therapists for symptoms measured in the present study was high.

It should be noted that, although the assessor at follow-up was blinded, the assessors at post treatment were not blinded to outcome.

We did not include a formal assessment of treatment fidelity or therapist competence through audio-taping of sessions because of resource constraints. However, therapists were regularly supervised, and all interventions used in this study were based on detailed manuals. Supervisors worked closely with therapists reviewing process notes and the products generated by the individuals or groups. 
We only assessed behavioral indicators of change, rather than including dietary restraint or attitudinal aspects of bulimic symptomatology, such as weight and shape. We omitted the latter, as they are more difficult to assess reliably (41).

A number of our subjects were receiving antidepressant medication. We would not necessarily view this as a limitation, but rather, given the current status of pharmacological treatment of bulimia nervosa, we would view this as a fair reflection of clinic cases. Importantly, there were no differences between groups in this respect.

We did not include a health economic assessment of patient's total service use during and after treatment and we do not know how many of them received additional treatment for their eating disorder and whether there was any difference in this respect between the groups. However, given that we are the only NHS treatment center in the area, we can reasonably assume that no other NHS or specialized service was obtained.

Finally, attrition rates were high but in keeping with those in relatively unselected clinic samples $(11,32,42)$. Future studies might want to conduct qualitative interviews to assess the reasons for dropout, but resource constraints prohibited that in the current study.

\section{Strengths of the Study}

Although the study had a number of limitations, it also had a fair number of strengths. The sample size was large. Discussion at meetings and on the list serve of the Eating Disorders Research Society continually points to the gap between empirically supported treatments and typical clinical practice (43). Our study took our patients as we found them in our NHS clinic and very few were excluded from the protocol. 
Approximately one third of patients in the study suffered from EDNOS. Roughly $40 \%$ of patients were on antidepressants. A similar proportion took illicit drugs and just $>20 \%$ drank alcohol $\geq 4$ times a week regularly, suggesting harmful use. Thus, a sizable subgroup of our patients would have been excluded from previous treatment trials $(9,44)$. Moreover, in contrast to typical efficacy trials, our therapists were "ordinary" health professionals with widely differing levels of professional training and therapy experience, who had received only a modest amount of additional training in the study interventions, rather than highly trained "research therapists." Thus, the study was conducted in an ecologically more "normal" environment than most other research trials in this area, and therefore fulfilled many of the elements of an effectiveness study.

The inclusion of a long follow-up is an asset. Previously, treatment studies have rarely reported outcomes beyond 6 months to 1 year. In addition, the manualization of all treatments enhances generalizability and ability to disseminate. Furthermore, the interventions extend traditional CBT in two promising areas, MET and the development of interpersonal competencies. Finally, data modeling addresses limitations resulting from attrition.

In their critique of treatment for BN two and a half decades since it first entered the Diagnostic and Statistical Manual of Mental Disorders, Mitchell and colleagues (45) state, "new approaches need to be piloted" as "the limits of current approaches are obvious." We believe our study offers additional direction for just such alternative treatments.

\section{Further Considerations}

The overall "dose" of treatment (n 12 sessions) was relatively modest compared with other CBT packages, which are typically at least 16 to 20 sessions. It may be that a longer 
treatment package would have had added advantages in terms of improving outcomes in this group with high levels of comorbidity. Nonetheless, many patients showed good levels of improvement at the end of treatment comparable to those found in other studies, including the recent work of Fairburn and colleagues (7) that offered an enhanced CBT package, which included attention to interpersonal behaviors and perfectionism running for a total of 20 weeks. The data do suggest that there may be more than one way to apply CBT in the treatment of $\mathrm{BN}$, and that there may be viable, less costly alternatives to solely individual care. It is possible that MET may serve as a valuable addition to the therapist's repertoire for cases that do not show a good initial response to CBT.

Padmal de Silva was a co-investigator but died before the study's publication. Padmal was born in Sri Lanka and trained as a clinical psychologist at the Maudsley Hospital, where he worked until his retirement. He is remembered as fantastic teacher and a wise and generous colleague with an acute sense of fun. 


\section{REFERENCES}

1. National Collaborating Centre for Mental Health: National Clinical Practice Guideline: eating disorders: core interventions in the treatment and management of anorexia nervosa, bulimia nervosa, and related eating disorders. National Institute for Health and Clinical Excellence. 2004. Available at http://www.nice.org.uk.

2. Fairburn CG, Marcus MD, Wilson GT. Cognitive behavior therapy for binge eating and bulimia nervosa: a comprehensive treatment manual. In: Fairburn CG, Wilson GT, editors. Binge Eating: Nature, Assessment and Treatment. New York: Guilford Publications; 1993.

3. Fairburn CG, Harrison P. Eating disorders. Lancet 2003;361:407-16.

4. Treasure JL, Ward A. A practical guide to the use of motivational interviewing in anorexia nervosa. Eur Eat Disord Rev 1997;5:102-14.

5. Palmer R, Birchall H, McGrain L, Sullivan V. Self-help for bulimic disorders: a randomized controlled trial comparing minimal guidance with face-to-face or telephone guidance. Br J Psychiatry 2002;181:230-5.

6. Mitchell JE, Crosby RD, Wonderlich SA, Crow S, Lancaster K, Simonich H, SwanKremeier L, Lysne C, Myers TC. A randomized trial comparing the efficacy of cognitivebehavioral therapy for bulimia nervosa delivered via telemedicine versus face-to-face. Behav Res Ther 2008;46:581-92. 
7. Fairburn CG, Cooper Z, Doll HA, O’Connor ME, Bohn K, Hawker DM, Wales JA, Palmer RL. Transdiagnostic cognitive-behavioral therapy for patients with eating disorders: a twosite trial with 60-week follow-up. Am J Psychiatry 2009;166:311-9.

8. Mitchell JE, Hoberman JE, Peterson CB, Mussell M, Pyle RL. Research on the psychotherapy of bulimia nervosa: half empty or half full. Int J Eat Disord 1996;220:219 -29.

9. Chen EY, Touyz SW, Beumont PJV, Fairburn CG, Griffiths R, Butow P, Russell J, Schotte DE, Gertler R, Basten C. Comparison of group and individual cognitive-behavioral therapy for patients with bulimia nervosa. Int J Eat Disord 2003;33:241-54.

10. Bailer U, de Zwaan M, Leisch F, Strnad A, Lennkh-Wolfsberg C, El-Giamal N, Hornik K, Kasper S. Guided self-help versus cognitive-behavioral group therapy in the treatment of bulimia nervosa. Int J Eat Disord 2004;35:522-37.

11. Nevonen L, Broberg AG. A comparison of sequenced individual and group psychotherapy for patients with bulimia nervosa. Int J Eat Disord 2006;39:117-27.

12. Steel Z, Jones J, Adcock S, Clancy R, Bridgford-West L, Austin J. Why the high rate of dropout from individualized cognitive-behavior therapy for bulimia nervosa? Int J Eat Disord 2000;28:209 -14.

13. Waller G, Schmidt U, Treasure J, Emmanueli F, Crockett J, Key A, Murphy B, Yeomans M, Alenya J, Murray K. Pathways to care and matching clients to treatments: an audit of 
tertiary level eating disorder services. London, UK: NHS Specialist Clinical Audit Programme for South London, Kent, Surrey and Sussex, 2005.

14. Hettema J, Steele J, Miller WR. Motivational interviewing. Annu Rev Clin Psychol 2005;1:91-111.

15. Dean, H, Touyz SW, Rieger E, Thornton CE. Group motivational enhancement therapy as an adjunct to inpatient treatment for eating disorders: a preliminary study. Eur Eat Disord $\operatorname{Rev} 2007 ; 16: 256-67$.

16. Dunn E, Neighbors C, Larimer M. Motivational enhancement therapy and self-help treatment for binge eaters. Psychol Addict Behav 2006;20:44-52.

17. Treasure J, Schmidt U. A Clinician's Guide to Getting Better Bit(e) by Bit(). Hove, East Sussex, United Kingdom: Psychology Press; 1997.

18. Cooper P. Bulimia Nervosa. London: Robinson; 1993.

19. Weiss L, Katzman M, Wolchik S. You Can’t Have Your Cake and Eat It Too: A Program for Controlling Bulimia. Phoenix, Arizona: Golden Psych Press; 1986.

20. Weiss L, Katzman M, Wolchik S. Treating bulimia: a psychoeducational approach. New York: Pergamon Press; 1985. 
21. Wolchik SA, Weiss L, Katzman MA. An empirically validated, short-term psychoeducational group treatment program for bulimia. Int J Eat Disord 1986;5:21-34.

22. Kordy H, Richard M, Herrmann A, Murphy F, Treasure J, Charpentier P. A computer assisted eating disorder specific quality management system: EQUAL-TREAT. Eur Eat Disord Rev 1999;7:239 -58.

23. Cooper Z, Fairburn CG. The eating disorders examination. In: Fairburn CG, Wilson GT, editors. Binge eating: nature, assessment and treatment. 12th ed. New York: Guilford Press; 1993.

24. Keller MB, Lavari PW, Friedman B, Nielsen E, Endicott J, McDonald-Scott P, Andreasen NC. The longitudinal interval follow-up evaluation: a comprehensive method for assessment of outcome in prospective longitudinal studies. Arch Gen Psychiatry 1987;44:540-8.

25. Derogatis LR. Symptom Checklist-90. Baltimore: Johns Hopkins University Press; 1977.

26. Bauer S, Winn S, Schmidt U, Kordy H. Construction, scoring, and validation of the short evaluation of eating disorders (SEED). Eur Eat Disord Rev 2005;13:191-200.

27. Kächele H, Studiengruppe MZ-ESS. A multicentre study concerning expenditure and success in psychodynamically oriented therapy of eating disorders — study design and initial results. Psychotherapie, Psychosomatik und medizinische Psychologie 1999;49:100-8. 
28. Kächele H, Kordy H, Richard M, Research Group TR-EAT. Therapy amount and outcome of inpatient psychodynamic treatment of eating disorders in Germany: data from a multicenter study. Psych Res 2001; 11:239 -57.

29. McConnaughy EA, Prochaska JO, Velicer WF. Stages of change in psychotherapy: measurement and sample profiles. Psychotherapy: Theory, Research and Practice $1983 ; 20: 368-75$.

30. Rabe-Hesketh S, Pickles A, Taylor C. Reliable estimation of generalized linear mixed models using adaptive quadrature. Stata J 2002;2:1-21.

31. Hay PJ, Bacaltchuk J. Bulimia nervosa. Clin Evid 2006;15:1315-31.

32. Waller G. Drop-out and failure to engage in individual outpatient cognitive behavior therapy for bulimic disorders. Int J Eat Disord 1997;22:35-41.

33. Miller WR, Zweben A, DiClemente CC, Rychtarik R. Motivational enhancement manual: a clinical research guide for therapists treating individuals with alcohol abuse and dependence. Project MATCH Monograph Series. Vol 2. Rockville, MD: National Institute of Alcohol Abuse and Alcoholism; 2002.

34. Agras WS, Crow SJ, Halmi KA, Mitchell JE, Wilson GT, Kraemer HC. Outcome predictors for the cognitive behavior treatment of bulimia nervosa: data from a multisite study. Am J Psychiatry 2000;157:1302- 8. 
35. Treasure JL, Katzman M, Schmidt U, Troop N, Todd G, de Silva P. Engagement and outcome in the treatment of bulimia nervosa: first phase of a sequential design comparing motivation enhancement therapy and cognitive behavioural therapy. Behav Res Ther 1999;37:405-18.

36. Miller WR, Rose GS. Toward a theory of motivational interviewing. Am Psychol 2009;64:527-37.

37. Olmsted MP, Davis R, Garner DM, Eagle M, Rockert W, Irvine MJ. Efficacy of a brief psychoeducational intervention for bulimia nervosa. Behav Res Ther 1991;29:71- 83.

38. Fairburn CG, Cooper Z, Shafran R. Cognitive behavior therapy for eating disorders: a "transdiagnostic" theory and treatment. Behav Res Ther 2003;41:509 -28.

39. Treasure J, Schmidt U, Troop N, Tiller J, Todd G, Keilen M, Dodge E. First step in the management of bulimia nervosa: controlled trial of a therapeutic manual. BMJ 1994;308:686 -9 .

40. Fairburn CG, Beglin SJ. Assessment of eating disorders: interview or self-report questionnaire? Int J Eat Disord 1994;16:363-70.

41. Mond JM, Hay PJ, Rodgers B, Owen C. Self-report versus interview assessment of purging in a community sample of women. Eur Eat Disord Rev 2007;15:403-9. 
42. Mahon J, Bradley SN, Harvey PK, Winston AP, Palmer RL. Childhood trauma has doseeffect relationship with dropping out from psychotherapeutic treatment for bulimia nervosa: a replication. Int J Eat Disord 2001;30:138-48.

43. Eating Disorders Research Society List Serve exchange, March 2, 2009.

44. Mitchell JE, Maki DD, Adson DE, Ruskin BS, Crow S. The selectivity of inclusion and exclusion criteria in bulimia nervosa treatment studies. Int J Eat Disord 1997;22:243-52.

45. Mitchell, JE, Agras, S; Wonderlich, S. Treatment of bulimia nervosa: where are we now and where are we going? Int J Eat Disord 2006;36:1-6. 
Table 1. Baseline data

\begin{tabular}{llllll}
\hline & Total $\mathrm{n}=225$ & MET-I $\mathrm{n}=79$ & MET-G n $=73$ & CBT-G n $=73$ & P* \\
& Mean (SD) & Mean (SD) & Mean (SD) & Mean (SD) & \\
\hline Age & $29.3(7.5)$ & $31.0(7.7)$ & $28.9(8.1)$ & $27.8(6.3)$ & .02 \\
BMI & $24.7(7.6)$ & $25.1(7.7)$ & $23.5(5.9)$ & $25.5(8.9)$ & .06 \\
Binges $^{\mathrm{a}}$ & $3.6(1.4)$ & $3.5(1.5)$ & $3.7(1.4)$ & $3.6(1.4)$ & .03 \\
Vomiting $^{\mathrm{a}}$ & $3.4(1.7)$ & $3.3(1.7)$ & $3.7(1.6)$ & $3.3(1.6)$ & .03 \\
Laxatives $^{\mathrm{a}}$ & $1.8(1.6)$ & $1.9(1.4)$ & $1.8(1.4)$ & $1.7(1.3)$ & .04 \\
\hline
\end{tabular}

a Coding frequency: " $1=$ not at all"; " $2=$ up to $1 \times$ week"; " $3=2$ to $3 \times$ week"; " $4=4 \times$ week up to daily"; "5 = more $1 \times$ day."

*One-way analysis of variance, $\mathrm{p}$ value.

MET-I = individual course of motivational enhancement therapy offered before individual cognitive therapy; MET-G = individual motivational enhancement therapy offered before cognitive behavior therapy group; CBT-G = individual cognitive behavior therapy offered before cognitive behavior therapy group; $\mathrm{SD}=$ standard deviation; $\mathrm{BMI}=$ body mass index. 
Table 2

\begin{tabular}{lllllll}
\hline Group & Behavior & $\begin{array}{l}\text { Assessment } \\
\%(\mathrm{n})\end{array}$ & $\begin{array}{l}\text { Week } 4 \\
\%(\mathrm{n})\end{array}$ & $\begin{array}{l}\text { End of } \\
\text { treatment } \\
\%(\mathrm{n})\end{array}$ & $\begin{array}{l}\text { 1-year } \\
\text { follow-up } \\
\%(\mathrm{n})\end{array}$ & $\begin{array}{l}2.5 \text {-year } \\
\text { follow-up } \\
\%(\mathrm{n})\end{array}$ \\
\hline MET-I & Binge eating & $0.0(39)$ & $6.1(34)$ & $25.0(20)$ & $57.7(26)$ & $40.0(20)$ \\
& Vomiting & $17.8(45)$ & $32.3(34)$ & $25.0(20)$ & $57.7(26)$ & $40.0(20)$ \\
& Laxative use & $66.7(39)$ & $71.0(31)$ & $72.2(18)$ & $71.0(31)$ & $81.8(22)$ \\
\hline MET-G & Binge eating & $2.7(37)$ & $3.0(30)$ & $24.2(33)$ & $45.2(31)$ & $38.5(13)$ \\
& Vomiting & $16.7(36)$ & $26.7(30)$ & $24.2(33)$ & $45.2(19)$ & $38.5(21)$ \\
& Laxative use & $54.8(31)$ & $89.3(28)$ & $71.4(28)$ & $89.3(28)$ & $92.3(13)$ \\
\hline CBT-G & Binge eating & $5.0(40)$ & $12.1(36)$ & $40.0(20)$ & $63.2(19)$ & $57.2(21)$ \\
& Vomiting & $26.7(45)$ & $44.4(36)$ & $40.0(20)$ & $63.2(19)$ & $57.1(21)$ \\
& Laxative use & $53.7(41)$ & $74.3(35)$ & $82.4(17)$ & $74.3(35)$ & $84.2(19)$ \\
& & & & & &
\end{tabular}

MET-I = individual course of motivational enhancement therapy offered before individual cognitive therapy; MET-G = individual motivational enhancement therapy offered before cognitive behavior therapy group; CBT-G = individual cognitive behavior therapy offered before cognitive behavior therapy group; $\mathrm{SD}=$ standard deviation; $\mathrm{BMI}=$ body mass index. There was no significant group by time interaction on any of the behavioural variables. 
Table 3. Overall abstinence ratings over time for patient and therapist

\begin{tabular}{|c|c|c|c|c|}
\hline & $\begin{array}{l}\text { Baseline } \\
\%(\mathrm{n})\end{array}$ & $\begin{array}{l}\text { End of treatment } \\
\%(\mathrm{n})\end{array}$ & $\begin{array}{l}\text { 1-year follow-up } \\
\%(\mathrm{n})\end{array}$ & $\begin{array}{l}\text { 1.5-year follow- } \\
\text { up } \\
\% \text { (n) }\end{array}$ \\
\hline \multicolumn{5}{|l|}{ Bingeing } \\
\hline Patient & $2.5(38)$ & $29(32)$ & $40(22)$ & $46(17)$ \\
\hline Therapist & $12.5(70)$ & $46(18)$ & N/A & N/A \\
\hline \multicolumn{5}{|l|}{ Vomiting } \\
\hline Patient & $21(45)$ & $29(35)$ & $54(23)$ & $46(20)$ \\
\hline Therapist & $23(72)$ & $45(37)$ & N/A & N/A \\
\hline \multicolumn{5}{|l|}{ Laxative use } \\
\hline Patient & $59(40)$ & $75(33)$ & $80(33)$ & $85(16)$ \\
\hline Therapist & $67(66)$ & $87(35)$ & N/A & N/A \\
\hline
\end{tabular}

N/A = not applicable 
Figure 1. Consort diagram

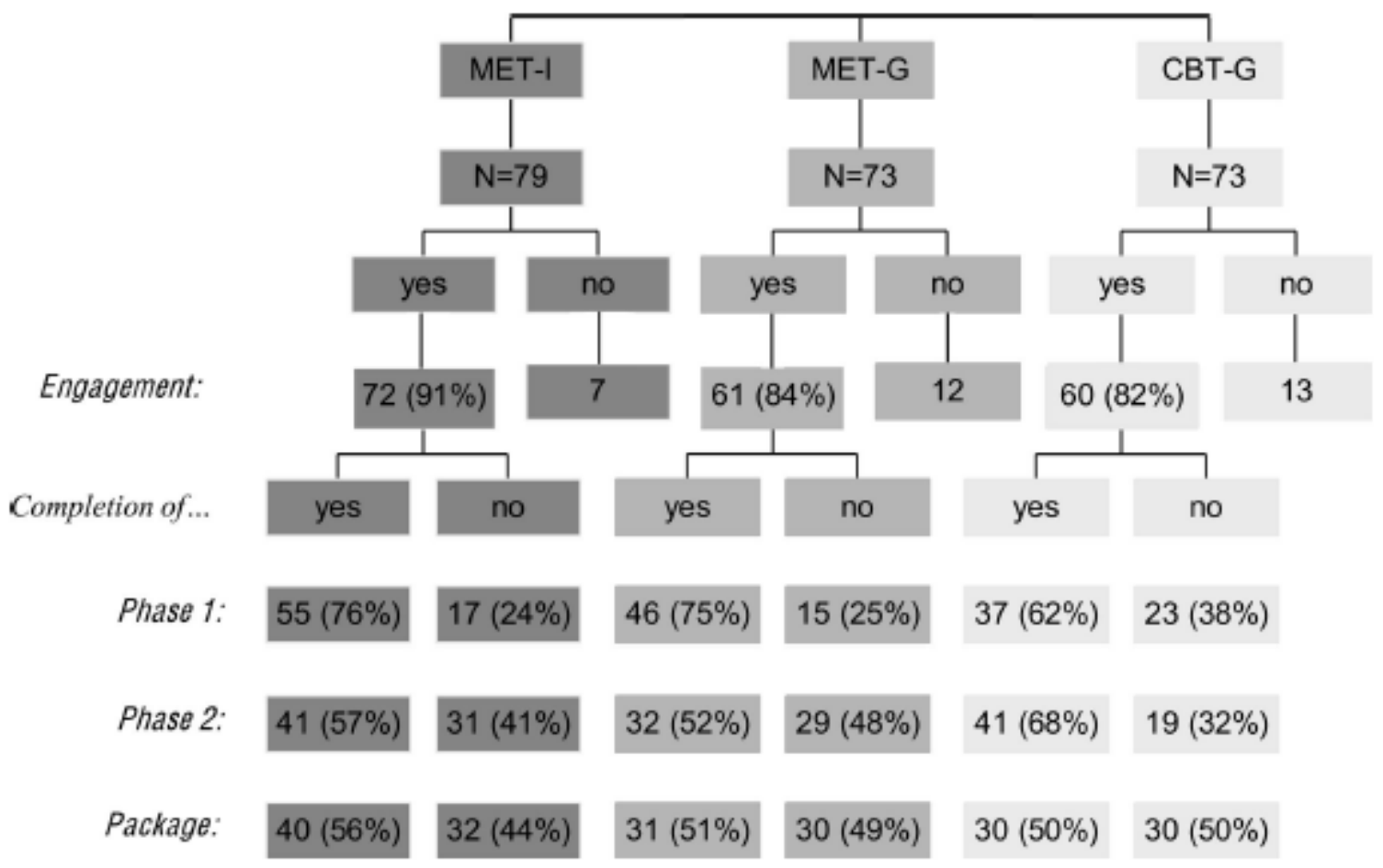

MET-I = individual course of motivational enhancement therapy offered before individual cognitive therapy; MET-G = individual motivational enhancement therapy offered before cognitive behavior therapy group; CBT-G = individual cognitive behavior therapy offered before cognitive behavior therapy group. 
Figure 2. Treatment dropout by condition

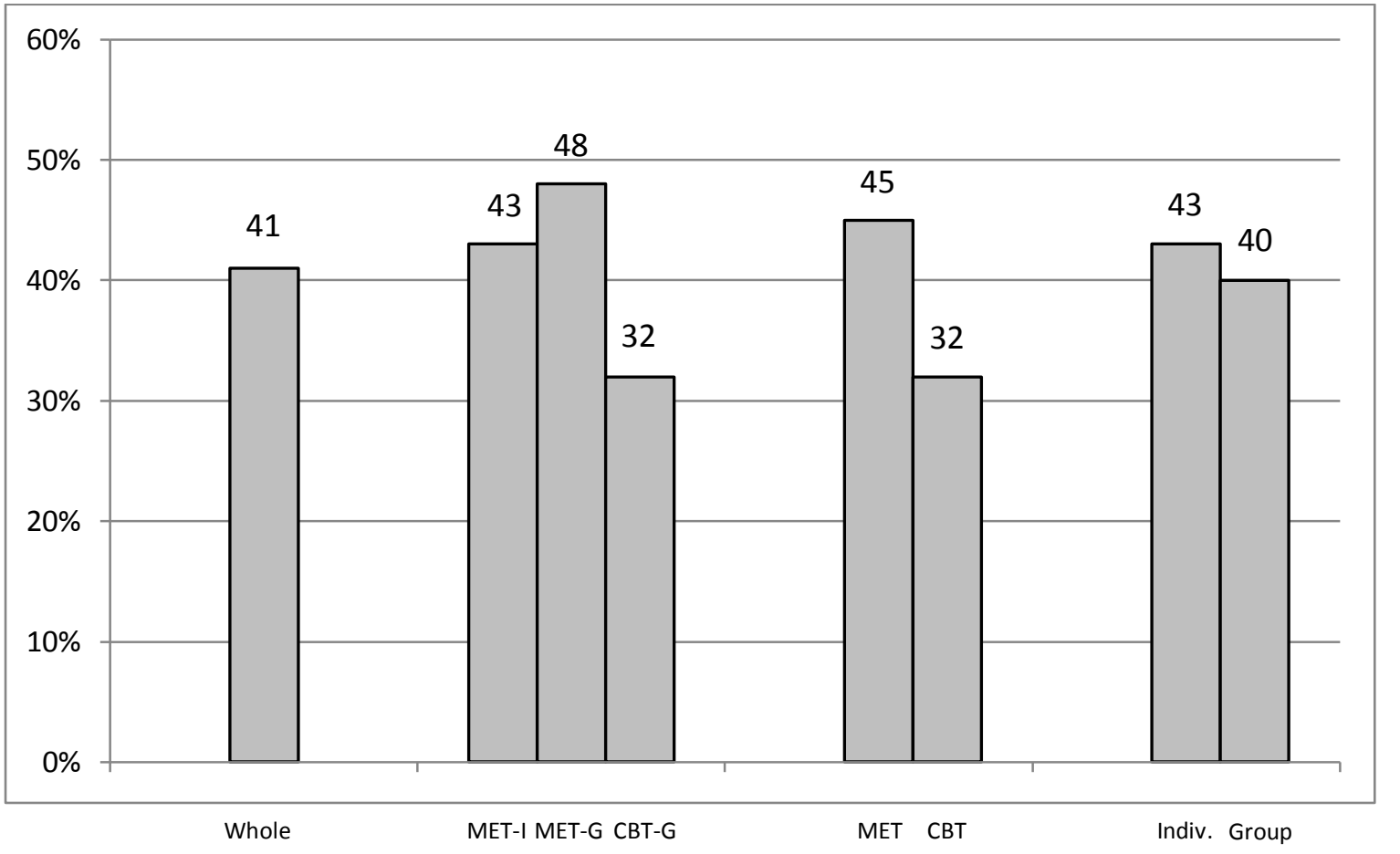

There were no significant differences between groups $(\mathrm{p}=.08)$. MET-I $=$ individual course of motivational enhancement therapy offered before individual cognitive therapy; MET-G = individual motivational enhancement therapy offered before cognitive behavior therapy group; CBT-G = individual cognitive behavior therapy offered before cognitive behavior therapy group; $\mathrm{MET}=$ motivational enhancement therapy; $\mathrm{CBT}=$ cognitive-behavioral therapy. 